ARTICLE OPEN

\title{
Thermal transport in layer-by-layer assembled polycrystalline graphene films
}

David Estrada ${ }^{1,2,3}$, Zuanyi Li ${ }^{1,4,5}$, Gyung-Min Choi ${ }^{6,7,8}$, Simon N. Dunham ${ }^{6,7,9}$, Andrey Serov ${ }^{1,2}$, Jungchul Lee ${ }^{10,11}$, Yifei Meng ${ }^{6,7}$, Feifei Lian ${ }^{1,2,5}$, Ning C. Wang ${ }^{1,2,5}$, Alondra Perez ${ }^{12}$, Richard T. Haasch ${ }^{6}$, Jian-Min Zuo ${ }^{6,7}$, William P. King ${ }^{1,10,13}$, John A. Rogers (D) ${ }^{1,6,7,13,14}$, David G. Cahill ${ }^{6,7}$ and Eric Pop (D) $^{5,15}$

New technologies are emerging which allow us to manipulate and assemble 2-dimensional (2D) building blocks, such as graphene, into synthetic van der Waals (vdW) solids. Assembly of such vdW solids has enabled novel electronic devices and could lead to control over anisotropic thermal properties through tuning of inter-layer coupling and phonon scattering. Here we report the systematic control of heat flow in graphene-based vdW solids assembled in a layer-by-layer (LBL) fashion. In-plane thermal measurements (between $100 \mathrm{~K}$ and $400 \mathrm{~K}$ ) reveal substrate and grain boundary scattering limit thermal transport in vdW solids composed of one to four transferred layers of graphene grown by chemical vapor deposition (CVD). Such films have room temperature in-plane thermal conductivity of $\sim 400 \mathrm{Wm}^{-1} \mathrm{~K}^{-1}$. Cross-plane thermal conductance approaches $15 \mathrm{MWm}^{-2} \mathrm{~K}^{-1}$ for graphene-based vdW solids composed of seven layers of graphene films grown by CVD, likely limited by rotational mismatch between layers and trapped particulates remnant from graphene transfer processes. Our results provide fundamental insight into the in-plane and cross-plane heat carrying properties of substrate-supported synthetic vdW solids, with important implications for emerging devices made from artificially stacked 2D materials.

npj 2D Materials and Applications (2019)3:10; https://doi.org/10.1038/s41699-019-0092-8

\section{INTRODUCTION}

The past decade of graphene research has accelerated scientific discovery of $2 \mathrm{D}$ transition metal dichalcogenides, ${ }^{1,2}$ phosphorene, ${ }^{3}$ silicene, ${ }^{4}$ and 2D hexagonal boron nitride. ${ }^{5}$ These materials have unique electrical, thermal, optical, and mechanical properties as compared to their 3-dimensional (3D) counterparts. Electrically, such 2D building blocks exhibit metallic, semiconducting, and insulating behavior, providing novel material combinations for electronic device design. ${ }^{1,6}$ For example, LBL assembly of graphene with other $2 \mathrm{D}$ materials has resulted in ultrathin heterostructures suitable for tunneling field effect transistors ${ }^{7-9}$ and ultrathin optoelectronic devices. ${ }^{10,11}$ However, the thermal properties of LBL assembled artificial vdW solids have received less attention. Similar to naturally occurring vdW solids, artificial vdW solids are expected to have strong in-plane bonds and weak inter-layer vdW interactions, resulting in anisotropic thermal properties between the in-plane and cross-plane directions. ${ }^{12-14}$

In this work, we use a combination of suspended-bridge electrical thermometry and time-domain thermoreflectance (TDTR) to probe heat flow in LBL assembled graphene-based vdW solids. We pay particular attention to the role of external influences on thermal transport in such films, e.g., grain size and the role of the substrate. We find the in-plane thermal conductivity $\left(k_{\|}\right)$of our substrate-supported CVD-grown polycrystalline graphene is approximately equal to that of substratesupported exfoliated graphene ${ }^{15}$ at low temperatures $\left(\approx 120 \mathrm{Wm}^{-1} \mathrm{~K}^{-1}\right.$ at $\left.100 \mathrm{~K}\right)$. The $k_{\|}$peaks at around room temperature between 300 and $400 \mathrm{Wm}^{-1} \mathrm{~K}^{-1}$, depending on the grain size $\left(L_{\mathrm{g}}\right)$, but independent of the number of transferred graphene films grown by CVD which we denote as N. Importantly, we find the $k_{\|}$of substrate-supported polycrystalline graphene with $L_{\mathrm{g}} \sim 140 \mathrm{~nm}$ is approximately $70 \%$ of substrate-supported exfoliated graphene, consistent with our previous theoretical study. ${ }^{16}$ This is an order of magnitude less than freely suspended graphene $\left(2000-4000 \mathrm{Wm}^{-1} \mathrm{~K}^{-1}\right){ }^{13,14,17-19}$ and consistent with previous electrical thermometry ${ }^{15}$ and Raman thermometry ${ }^{20}$ measurements of $\mathrm{SiO}_{2}$-supported graphene. Our results highlight the important roles that substrate and grain boundary scattering play for in-plane thermal transport properties of ultra-thin LBL assembled graphene vdW solids $(N=1-4)$.

We also probe the cross-plane thermal conductance $\left(G_{\perp}\right)$ of $L B L$ assembled graphene vdW solids and find that it is consistently below that of A-B stacked few-layer graphene. These findings present data for the grain size effect on in-plane thermal transport,

\footnotetext{
${ }^{1}$ Micro \& Nanotechnology Laboratory, University of Illinois, Urbana-Champaign, IL 61801, USA; ${ }^{2}$ Department of Electrical \& Computer Engineering, University of Illinois, UrbanaChampaign, IL 61801, USA; ${ }^{3}$ Micron School of Materials Science \& Engineering, Boise State University, Boise, ID 83725, USA; ${ }^{4}$ Department of Physics, University of Illinois, Urbana-

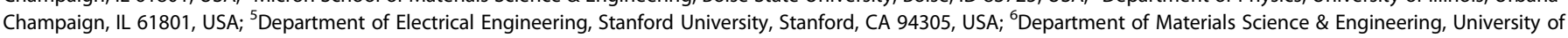
Illinois, Urbana-Champaign, IL 61801, USA; ${ }^{7}$ Fredrick Seitz Materials Research Laboratory, University of Illinois, Urbana-Champaign, IL 61801, USA; ${ }^{8}$ Department of Energy Science,

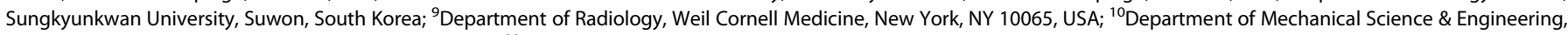
University of Illinois, Urbana-Champaign, IL 61801, USA; ${ }^{11}$ Department of Mechanical Engineering, Korean Advanced Institute of Science and Technology, Daejeon, South Korea; ${ }^{12}$ Department of Mechanical Engineering, Boise State University, Boise, ID 83725, USA; ${ }^{13}$ Beckman Institute, University of Illinois, Urbana-Champaign, IL 61801, USA; ${ }^{14}$ Department

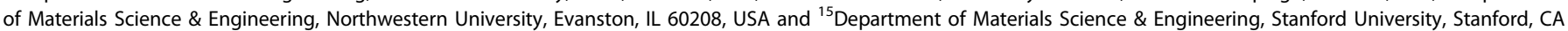
94305, USA

Correspondence: David Estrada (daveestrada@boisestate.edu) or Eric Pop (epop@stanford.edu)
}

Received: 6 August 2018 Accepted: 31 January 2019

Published online: 05 March 2019 
a

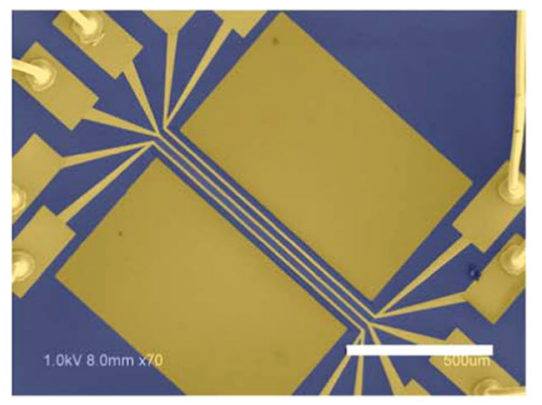

d

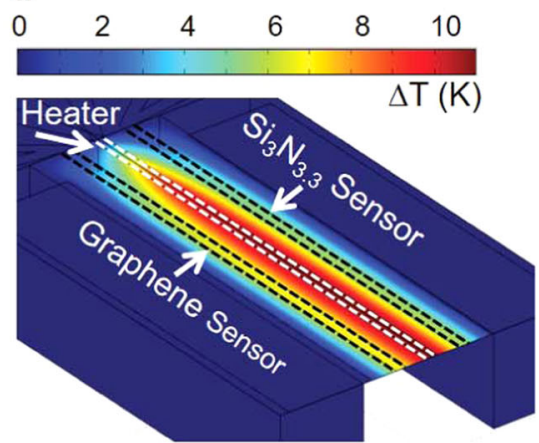

b

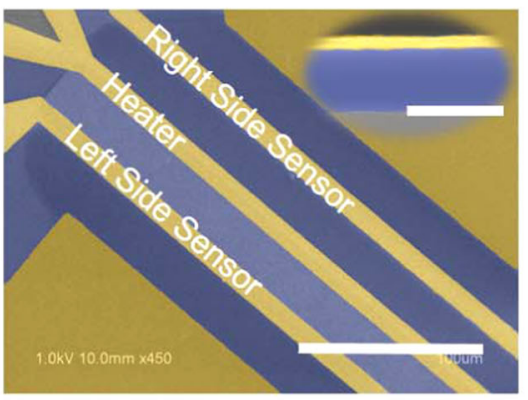

e

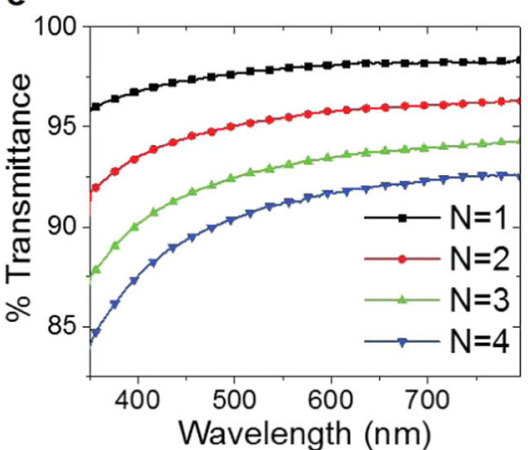

C

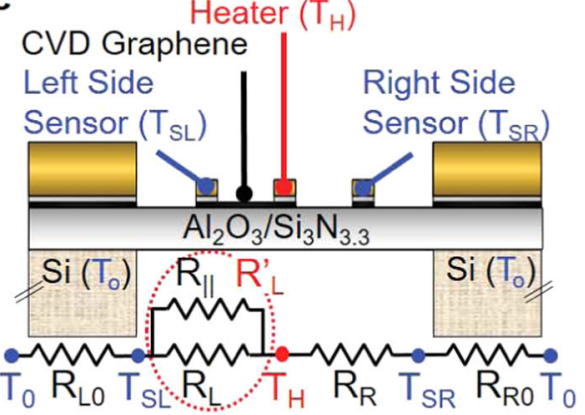

f

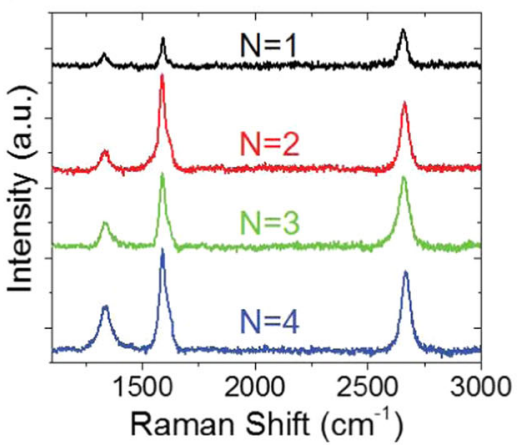

Fig. 1 In-plane thermal measurement platform and graphene characterization. a SEM image of differential electrical thermometry platform showing wire bonds for electrical access to heater and sensors. Scale bar is $500 \mu \mathrm{m}$. b Higher magnification SEM image showing suspended region of the platform with graphene patterned between the center heater and left sensor. Scale bar is $100 \mu \mathrm{m}$. The inset shows a crosssection SEM image of the membrane thickness (purple color) capped by a thin metal layer (gold color). Scale bar is $300 \mathrm{~nm}$. c Schematic of suspended thermometry platform. The thermal circuit is shown below. The left side sensor measures the heat flow through the graphene side of the sample while the right side sensor measures the heat flow through the right side silicon nitride membrane. $\mathbf{d}$ Temperature distribution in a 3D finite element simulation of the experimental test structure with a single transferred layer of polycrystalline graphene and power applied to the center heater. e, $\mathbf{f}$ Transmittance and Raman characteristics of layer-by-layer assembled graphene films

as well as data probing cross-plane thermal transport in polycrystalline LBL assembled graphene vdW solids supported by dielectric substrates. Our results are highly relevant for future LBL assembled devices and interconnects, highlighting an approach which may be used to tune the heat flow properties of LBL assembled 2D heterostructures.

\section{RESULTS}

In-plane thermal measurements

Figure 1a-c shows scanning electron microcroscopy (SEM) images and a schematic cross-section of the suspended thermometry bridges used in this study. The layered graphene samples are supported by a thin silicon nitride bridge to provide thermal isolation and mechanical robustness. X-ray photoelectron spectroscopy reveals a $\mathrm{Si}_{3} \mathrm{~N}_{3.3}$ stochiometry of the supporting bridges (Supplementary Information) and their thickness varies between 150 and $300 \mathrm{~nm}$ depending on the fabrication run (measured by ellipsometry and compared to cross-sectional SEM, inset of Fig. 1b). Polycrystalline graphene is grown on copper foils purchased from Alfa Aesar (CAS 7440-50-8) and then transferred to the $\mathrm{Si}_{3} \mathrm{~N}_{3.3}$, (initially supported on $\mathrm{Si}$ ) through a wet-transfer process using a polymer scaffold. ${ }^{21-24}$ The polymer is removed in organic solvents followed by annealing in $\mathrm{Ar} / \mathrm{H}_{2}$ to remove residual contaminants (see Methods). For samples with more than a single transferred layer of CVD graphene the wet-transfer and anneal process is repeated in a LBL fashion to achieve artificial graphene vdW solids with up to $N=4$ CVD layers. Metal heater and sensor strips are then patterned on top by photolithography, separated from the graphene by a thin evaporated $\mathrm{SiO}_{2}$ layer which serves as electrical insulation (see Methods and Supplement). The wafer is back-etched to suspend the supporting $\mathrm{Si}_{3} \mathrm{~N}_{3.3}$ membrane (Fig. 1c). Our device yield is enhanced by utilizing a thin $\mathrm{Al}_{2} \mathrm{O}_{3}$ layer as a through-wafer etch stop for the $\mathrm{BOSCH} 2$ process. The contrast of the graphene and the suspended region of the membrane are easily distinguishable in the final test structure (Fig. 1b).

We use optical transmittance measurements and Raman spectroscopy to characterize the assembled graphene stacks. We find the optical transmittance at $550 \mathrm{~nm}$ decreases by $\sim 2.8 \%$ with each new layer, in good agreement with previous work (Fig. 1e). ${ }^{25}$ Raman analysis of LBL assembled graphene stacks shows a decreasing intensity ratio of 2D-peak to G-peak $\left(I_{2 \mathrm{D}} / I_{\mathrm{G}}\right)$, and increasing D-peak intensity with increasing transfers (Fig. 1f). From the D-peak in the Raman spectra we can estimate the grain size of a single transferred graphene film $(N=1)$ as $L_{\mathrm{g}}(\mathrm{nm})=2 \times 10^{-10}$ $\lambda^{4}\left(I_{D} / I_{G}\right)^{-1}$, where $\lambda$ is the excitation laser wavelength and $\left(I_{D} / I_{G}\right)$ is the D-peak to G-peak integrated intensity ratio. ${ }^{26,27}$ The CVD graphene used in the LBL assembly of graphene vdW solids has an average grain size of $L_{\mathrm{g}} \sim 140 \pm 80 \mathrm{~nm}$ (Supplementary Information). We note this is not necessarily a crystallite size defined by the distance between graphene grain boundaries, but rather the distance between Raman-active defects, including graphene wrinkles, $^{28}$ grain boundaries, ${ }^{29}$ transitions between single layer to bilayer thickness, ${ }^{30}$ and regions of polymer residue. ${ }^{31}$ Our measured $L_{\mathrm{g}}$ is also in good agreement with previous scanning transmission electron microscopy (STEM) imaging of polycrystalline graphene films grown by random nucleation using CVD on copper foils. $^{32}$

Electrical thermometry measurements proceed as follows. A heating power ( $\sim 5$ to $175 \mu \mathrm{W}$ ) is passed through the heater 
electrode, while the temperature is sensed by monitoring calibrated changes in the electrical resistance of the heater and two sensors. One side of the measurement platform provides the total in-plane thermal conductance $\left(G_{L}{ }^{\prime}\right)$ of the graphene and silicon nitride film, while the other measures only the supporting silicon nitride film $\left(G_{R}\right)$ (Fig. 1c and Fig. S9). The in-plane thermal conductance of the graphene layer(s) $\left(G_{\|}\right)$is thus obtained by subtraction. Heat flow measurements are performed from 100 to $400 \mathrm{~K}$ under vacuum $\left(\sim 10^{-5}\right.$ Torr $)$ where heat loss due to convection is neglible. The maximum heat loss due to radiation is $Q_{\text {rad }} \approx 1 \%$ at $400 \mathrm{~K}$, where $Q_{\text {rad }}=\sigma \epsilon A_{s}\left(T^{4}-T_{0}^{4}\right)$. Here, $\sigma$ is the Stefan-Boltzmann constant, $\epsilon$ is the membrane emmissivity (assumed to be 1 to provide an upper bound), $A_{\mathrm{s}}$ is the area of the suspended membrane, $T_{0}$ is the background temperature, and $T$ is the average temperature of the suspended membrane. We do not notice a significant temperature hysteresis in our measurements with increasing and decreasing ambient temperature sweeps. In addition, we have also compared a single transferred layer of CVD graphene to LBL assembled stacks of CVD graphene, and to non-equilibrium Green's function (NEGF) calculations, ${ }^{16}$ in order to elucidate the role of the supporting substrate and that of graphene grain boundaries on the films' thermal properties.

Similar to previous work from our group, we use a commercial software package (COMSOL Multiphysics) to extract the thermal properties of graphene from the electrical thermometry data. ${ }^{33,34}$ Figure $1 \mathrm{~d}$ shows our optimized 3D finite element method (FEM) model of the suspended thermometry platform and a typical extracted steady-state temperature profile when a graphene film is placed on one side of the platform (also see Supplementary Information). The simulation is performed using isothermal boundary conditions at the bottom and side surfaces of the platform (i.e., at the Si heat sink), while the symmetry plane and the surfaces of the supporting membrane, electrodes, and graphene are given adiabatic boundary conditions. Importantly, the 3D simulations include thermal contact resistance effects of all interfaces $^{33}$ (Supplementary Information), although these have a minimal effect on the extracted values of $k_{\| \mid}$. A constant power is applied to the center heater electrode, consistent with the Joule heating $\left(P_{\mathrm{H}}\right)$ induced in the measurements, and the structure is allowed to come to steady state. We then fit the simulated temperature rises in the heater and two sensors to the measured experimental data $\left(\Delta T_{\mathrm{H}}, \Delta T_{\mathrm{SL}}, \Delta T_{\mathrm{SR}}\right)$, using the thermal conductivity of the membrane $\left(k_{\mathrm{SiN}}\right)$ and graphene $\left(k_{\|}\right)$as fitting parameters. We find that although our suspended membrane geometry allows us to approximate 1-dimensional heat flow, approximately $10 \%$ of the heat flows in a 2D manner near the membrane edges. This is also in good agreement with our analytical model described below.

Analytically, the $k_{\|}$can be written as

$k_{\|}=G_{\|} \frac{L_{\mathrm{HL}}}{W h_{\mathrm{g}}}$,

where $G_{\|}$is the thermal conductance of the graphene, $L_{\mathrm{HL}}$ is the distance between the heater and graphene-side sensor, $W$ is the width of graphene, and $h_{\mathrm{g}}$ is the thickness of the graphene sample which is assumed to be $0.34 \mathrm{~nm}$ per transferred layer. This assumption provides an upper bound on the extracted thermal conductivity, and small thickness fluctuations (e.g., bilayer regions) are not expected to affect heat flow in otherwise continuous single layer graphene. ${ }^{35} G_{\|}$is calculated by subtracting the $\mathrm{Si}_{3} \mathrm{~N}_{3.3}$ thermal conductance $\left(G_{L}\right)$ from the combined thermal conductance of the $\mathrm{Si}_{3} \mathrm{~N}_{3.3}$ and graphene $\left(G_{L}^{\prime}\right)$ measured in our differential setup as follows:

$G_{\|}=G_{\mathrm{L}}^{\prime}-G_{\mathrm{L}}=\frac{P_{\mathrm{H}}(1-a) \beta}{\Delta T_{\mathrm{H}}-\Delta T_{\mathrm{SL}}}-\frac{P_{\mathrm{H}} \alpha \beta}{\Delta T_{\mathrm{H}}-\Delta T_{\mathrm{SR}}} \frac{L_{\mathrm{HR}}}{L_{\mathrm{HL}}}$

Here, $P_{\mathrm{H}}$ is the heater power, $a$ and $\beta$ are dimensionless parameters which account for the asymmetry in heat flow perpendicular to the electrodes and heat loss parallel to the electrodes, respectively. $\Delta T_{\mathrm{H}}, \Delta T_{\mathrm{SL}}$ and $\Delta T_{\mathrm{SR}}$, are the measured temperature rises in the heater, graphene-side (left), and $\mathrm{Si}_{3} \mathrm{~N}_{3.3^{-}}$ side (right) sensors, respectively. The distance between the heater and the $\mathrm{Si}_{3} \mathrm{~N}_{3.3}$-side sensor is $L_{\mathrm{HR}}$. We find the analytical model results are within $5 \%$ of the values obtained by the more computationally expensive FEM model (Fig. 2a, b, and Supplementary Figs. S10 and S11), highlighting the advantage of our differential electrical thermometry platform in simplifying the thermal analysis (see Supplementary Information for additional details of the analytical model).

We find the thermal conductivity of our $\mathrm{Si}_{3} \mathrm{~N}_{3.3}$ films, deposited by plasma enhanced CVD is $\sim 1.3 \mathrm{Wm}^{-1} \mathrm{~K}^{-1}$ at $300 \mathrm{~K}$ and exhibits excellent agreement with data from the literature over the full temperature range of our measurements (Supplementary Figs. S11 e-h), providing a good control on our methods. The extracted in-plane thermal conductance values for our $N=1$ sample are shown in Fig. 2a. It is easily seen that the $G_{L}^{\prime}$ is significantly higher than $G_{L}$ due to the addition of a single layer of CVD graphene. We find the $G_{\|}$of substrate-supported polycrystalline graphene films increases almost linearly with each additional layer added to the stack (Fig. 2b). When the $G_{\|}$of the LBL graphene vdW solids are converted to $k_{\|}$(Fig. 2c), however, we do not find a significant dependence on layer number up to $N=4$, consistent with previous measurements on supported exfoliated few-layer graphene. $^{36}$

We then compare single-layer graphene with different average grain sizes obtained arbitrarily from two different CVD growths in order to elucidate the role of line defects on thermal transport in substrate-supported graphene. In Fig. $2 d$, we plot the thermal conductivity of our two $N=1$ CVD graphene samples (one with larger $L_{g}$ is from Fig. 2c; the other with smaller $L_{g}$ is from Supplementary Fig. S12) vs. their average grain sizes at different temperatures, as well as $k_{\|}$of monocrystalline exfoliated graphene (limited by the sample width $\sim 2 \mu \mathrm{m}) .{ }^{15}$ It is shown that the thermal conductivity increases with increasing grain size, reaching $\approx 70 \%$ of the $k_{\|}$for substrate-supported monocrystalline exfoliated graphene and similar to that of bulk copper when $L_{\mathrm{g}} \approx 140 \mathrm{~nm}$. Importantly, the measured dependence of $k_{\|}$(symbols) on the grain size shows good agreement with our NEGF calculations ${ }^{16}$ for $k_{\|}$vs. $L_{g}$ (Fig. 2d), where the calculated $k_{\|}$is the sum of the individual transverse (TA), longitudinal (LA), and flexural acoustic (ZA) phonon modes (Supplementary Fig. S12c). Furthermore, as shown in Supplementary Fig. S12d, the best fits to the experimental data of $k_{\|}$vs. $T$ are obtained using grain sizes $\left(L_{g}\right)$ of 140 and $60 \mathrm{~nm}$, which are in excellent agreement with the grain sizes extracted by Raman spectroscopy (Supplementary Figs. S2 and S3).

Figure $2 \mathrm{f}$ shows a comparison of our data to the selected data from the literature. It illustrates the dominate role of the substrate scattering (Fig. 2e-left) in suppressing the thermal conductivity of substrate-supported graphene as compared to freely suspended graphene. Additional phonon scattering by graphene grain boundaries (Fig. 2e-right) can further reduce its thermal conductivity, and our data provide the temperature-dependent thermal conductivity for substrate-supported polycrystalline graphene. We notice that polycrystalline graphene with small domain sizes still greatly exceeds the thermal conductivity of narrow graphene nanoribbons, ${ }^{33}$ suggesting such films may be a viable technology for flexible and transparent heat spreaders with potential applications in the field of transparent and flexible electronics.

\section{Cross-plane thermal measurements}

We now turn our attention to cross-plane heat flow in LBL assembled graphene vdW solids (Fig. 3). We assemble artificial graphene vdW solids with the number of transferred CVD 
a
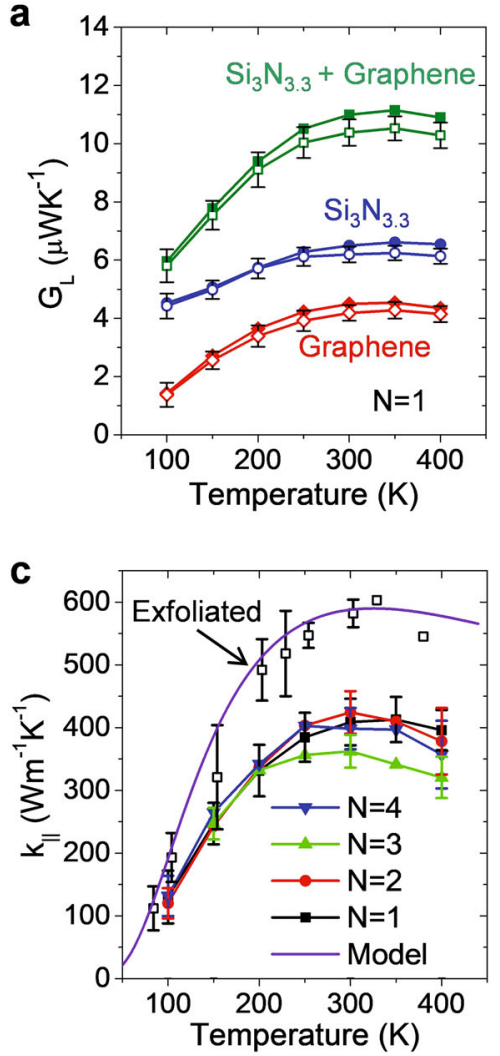

b
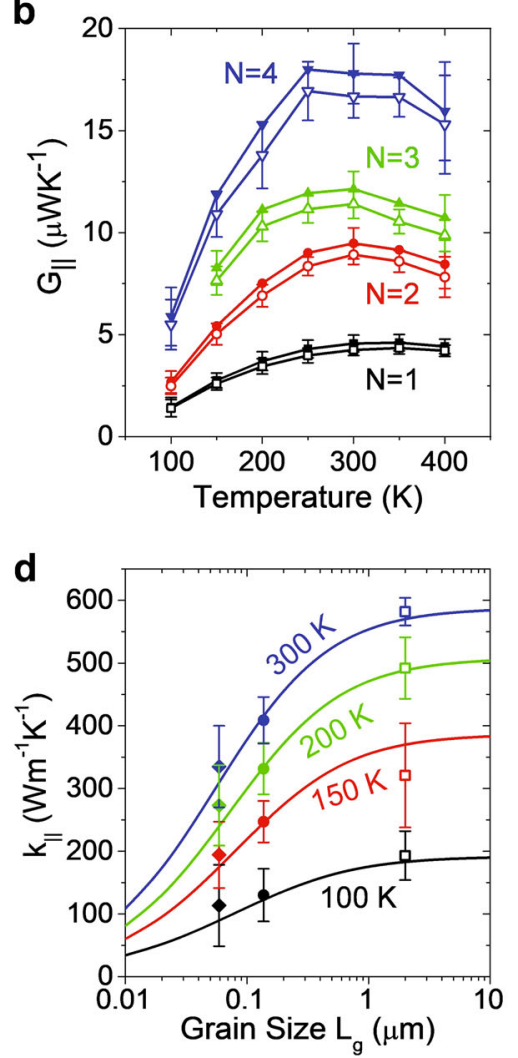

e
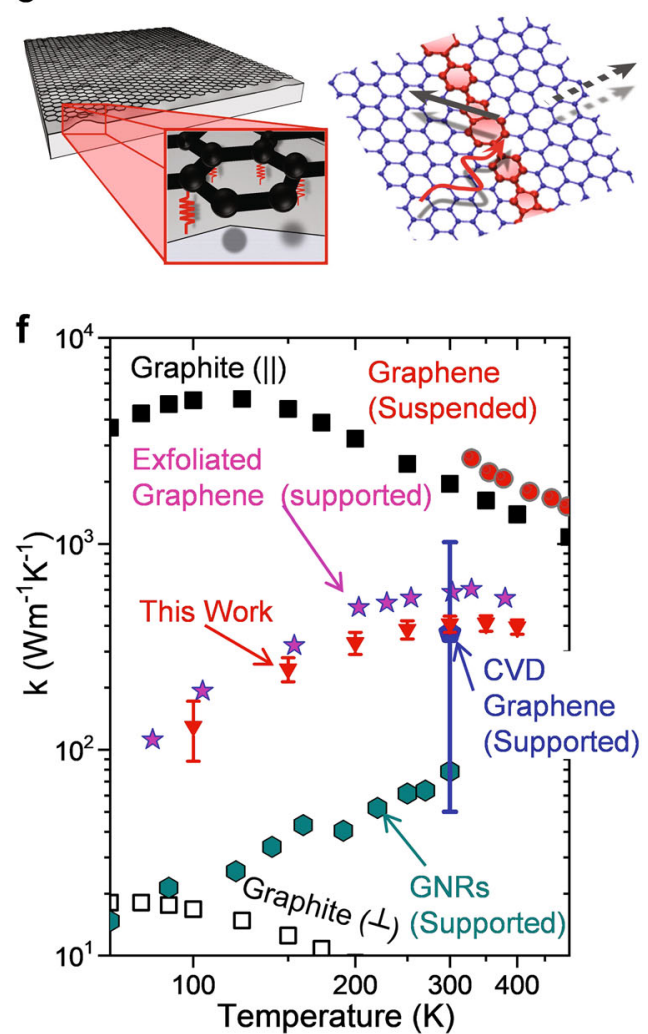

Fig. 2 In-plane thermal properties of layer-by-layer assembled graphene films. a Extracted thermal conductance of graphene and supporting silicon nitride membrane. b Thermal conductance of layer-by-layer assembled graphene films, adjusted to account for variations in sample length. In a, b solid-symbols are data extracted by 3D finite element modeling while open symbols are extracted using a simplified analytical model. c Calculated thermal conductivity from conductance in $\mathbf{b}$ compared to data for monocrystalline exfoliated graphene. ${ }^{15}$ The solid line through the exfoliated data is our calibrated NEGF model ${ }^{16}$ for monocrystalline graphene. ${ }^{15}$ d Thermal conductivity of two $N=1$ polycrystalline graphene samples with different average grain sizes of $60 \pm 30 \mathrm{~nm}$ and $140 \pm 80 \mathrm{~nm}$ by different growths (solid symbols) and data for exfoliated monocrystalline graphene (open squares), ${ }^{15}$ showing clear grain size dependences at different temperatures. Solid lines are obtained from NEGF calculations and show excellent agreement with the experimental data. e Schematic representation of substrate dampening and grain boundary scattering of graphene phonons. $\mathbf{f}$ Comparison of our polycrystalline graphene thermal conductivity to previous reports of suspended graphene, ${ }^{19}$ substrate-supported exfoliated graphene, ${ }^{15}$ metal-supported CVD graphene, ${ }^{43}$ graphene nanoribbons, ${ }^{33}$ and natural graphite. ${ }^{44}$ This work adds a "missing piece" to the literature highlighting the role of the substrate and grain boundary scattering in suppressing thermal conductivity in supported polycrystalline graphene over a wide temperature range. Error bars are the estimated experimental uncertainty (Supplementary Information)

graphene layers as $1 \leq N \leq 7$ on $\mathrm{SiO}_{2}$ substrates (Fig. $3 \mathrm{~d}$ ). We then deposit $\approx 80 \mathrm{~nm}$ of Al by shadow-mask evaporation in an electronbeam evaporator, and measure $G_{\perp}$ of the $\mathrm{Al} / \mathrm{N}$ layers of graphene/ $\mathrm{SiO}_{2}$ stack by TDTR, similar to previous work on exfoliated graphene samples. ${ }^{37}$ Figure $3 \mathrm{~b}$ shows TDTR results (symbols) and numerical solutions of our thermal model (solid lines) taking the measurements of the $\mathrm{Al} / \mathrm{SiO}_{2}$ interface as a reference. We find a reduction in $G_{\perp}$ with increasing layer number $N$. $G_{\perp}$ varies from $\approx 25$ to $15 \mathrm{MWm}^{-2} \mathrm{~K}^{-1}$ for $N=1-7$ (Fig. 3c). These values are consistently below those of A-B stacked exfoliated samples and approach a factor of two reduction in $G_{\perp}$ as compared to exfoliated graphene samples ${ }^{37}$ as $N$ approaches 7 transfers.

Figures $3 \mathrm{~d}-\mathrm{f}$ show the schematic representation of our stacked layers, a cross-sectional bright field STEM (BF-STEM) image with trapped particulates indicated in the dark contrast regions, and a line profile of image intensity across the stack, respectively. The peak intensities in Fig. $3 f$ illustrate the layered structure of our graphene stacks (Supplementary Fig. S13). High angle annular dark field STEM (HAADF-STEM) images and electron energy loss spectroscopy (EELS) analysis indicates the large trapped particulates are likely trapped copper particles, remnant from the etching and transfer process ${ }^{38,39}$ (Supplementary Fig. S13).

\section{DISCUSSION}

These measurements highlight the importance of material processing techniques on the structure-property correlations of thermal transport in LBL assembled graphene vdW solids. Our electrical thermometry measurements reveal the $k_{\|}$of $\mathrm{LBL}$ assembled graphene vdW solids is independent of the number of transferred graphene layers, up to $N=4$ transfers, with a value of $\sim 400 \mathrm{Wm}^{-1} \mathrm{~K}^{-1}$ at $T=300 \mathrm{~K}$ (Fig. 2c). Similar to mechanically exfoliated graphene supported by $\mathrm{SiO}_{2}$ substrates, ${ }^{15}$ we find the $k_{\|}$ of polycrystalline graphene films grown by CVD is greatly reduced by the supporting $\mathrm{Si}_{3} \mathrm{~N}_{3.3}$ substrates, due to suppression of the out-of-plane flexural mode (ZA) phonons. Residual polymer residue remnant from our microfabrication process could also contribute to scattering of ZA phonons and a further reduction of our polycrystalline graphene $k_{\|}{ }^{40}$ however it appears this effect is less than that of grain boundaries or the substrate here. Our results are also in good agreement with studies of encased graphene and ultra-thin graphite which indicated greater than 34 layers are needed to recover the $k_{\|}$of bulk graphite. ${ }^{36}$ Using Raman spectroscopy we correlated a reduction in $k_{\|}$to a reduction in the extracted $L_{g}$, a conclusion further supported by the study of Yasaei et al. which reported the detrimental effect of grain 
a

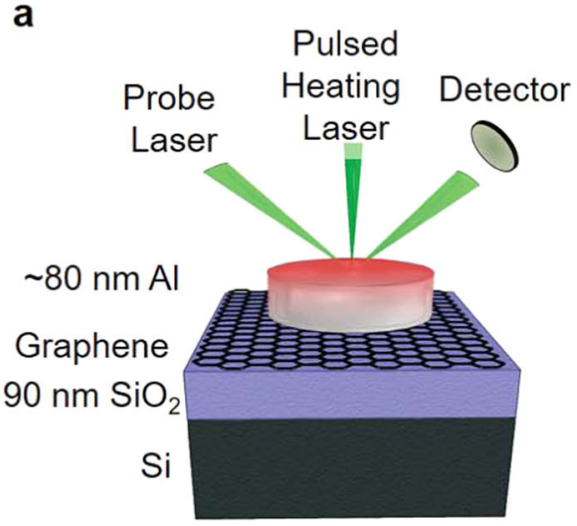

d

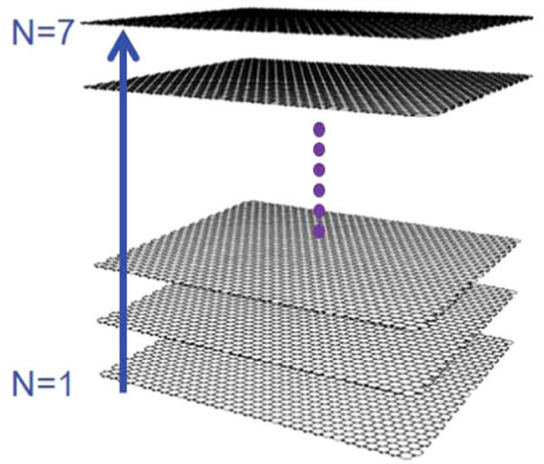

b 2

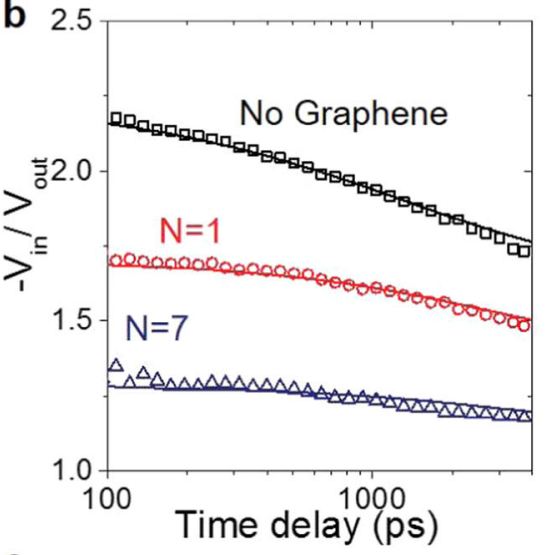

e

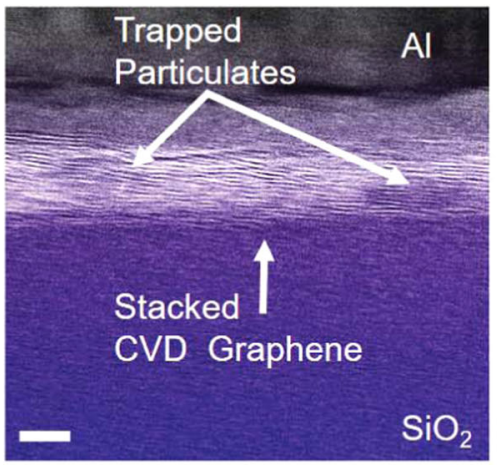

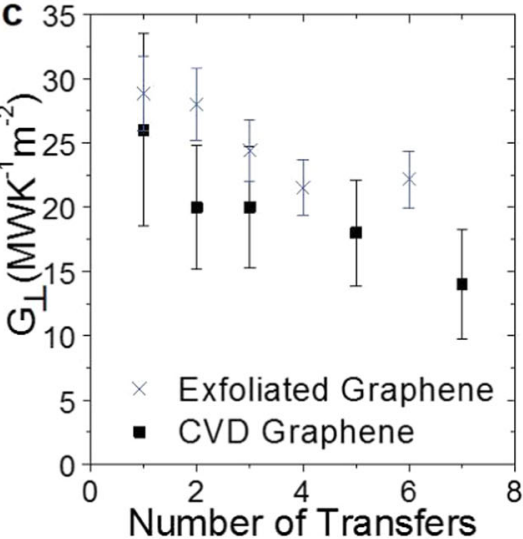

f

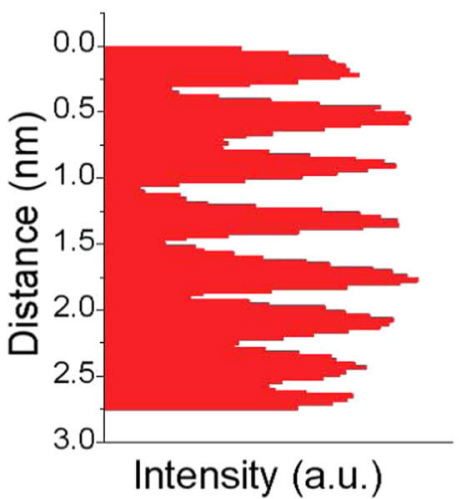

Fig. 3 Cross-plane thermal properties of layer-by-layer assembled graphene films. a Schematic representation of experimental setup for TDTR measurements on layer-by-layer assembled graphene films. b Ratio of the in-phase to out-of-phase signals as a function of delay time between pump and probe pulses. Representative measurements for $\mathrm{Al} / \mathrm{SiO}_{2} / \mathrm{Si}$ (no graphene), $\mathrm{Al} / \mathrm{graphene}(\mathrm{N}=1) / \mathrm{SiO} / \mathrm{Si}$, and $\mathrm{Al} / \mathrm{graphene}$ $(N=7) / \mathrm{SiO}_{2} / \mathrm{Si}$ are shown. The solid lines are best fits to the experimental data (open symbols). c Thermal conductance per unit area $\mathrm{G}_{\perp}$ of $\mathrm{Al}$ capped layer-by-layer assembled graphene films (filled squares) compared to single and few layer exfoliated graphene (x symbols). ${ }^{37}$ Error bars are the estimated experimental uncertainty. d, e Schematic representation and cross-section BF-STEM image of layer-by-layer assembled graphene films, respectively. The dark regions in e are trapped particulates believed to be Cu residues from the graphene transfer process. Scale bar is $5 \mathrm{~nm}$. $\mathbf{f}$ Intensity profile (BF-STEM) across the stack of a layer-by-layer assembled graphene film $(N=7)$ showing the carbon peak intensity correlating to different graphene layers in the stack. The additional eighth peak is attributed to a bilayer region in one of the CVD transferred films

boundaries on thermal transport depending on the grain boundary angle and morphology. ${ }^{41}$

Our cross-plane thermal conductance $\left(G_{\perp}\right)$ measurements of such LBL graphene films show a reduction in $G_{\perp}$ with increasing layer number $(N)$ from $G_{\perp} \approx 25$ to $15 \mathrm{MWm}^{-2} \mathrm{~K}^{-1}$ for $N=1$ to 7 . We attribute this reduction to a weakening of vdW coupling between layers, possibly induced by trapped particulates and a rotational mismatch in the lattices of the stacked graphene layers. However, we cannot exclude the possibility that $G_{\perp}$ is reduced due to reduced mechanical coupling to the underlying $\mathrm{SiO}_{2}$ resulting from a potential increase in sample stiffness or changes in the vibrational spectra as additional graphene layers are added to the stack. Importantly, as revealed by AFM and cross-section TEM analysis, residual contaminants from the polymer assisted transfer method remain on the surface and in between the CVD graphene layers even after annealing. When such CVD graphene films are assembled in a LBL fashion this can lead to reduced sample quality which must be taken into account when analyzing data for such LBL vdW solids. Hence, additional advancements in the synthesis of 2D based heterostructures and vdW solids are needed in order to probe truly fundamental thermal transport properties across pristine interfaces in such emerging materials.

In conclusion, we have probed the thermal transport of LBL assembled vdW solids constructed from individual graphene layers grown by CVD. Using electrical thermometry on suspended membrane platforms we measure the $k_{\|}$of such structures and find it varies between 120 and $425 \mathrm{Wm}^{-1} \mathrm{~K}^{-1}$ over the temperature range of $100-400 \mathrm{~K}$. This is nearly an order of magnitude lower than that of freely suspended CVD-grown graphene films and approximately $30 \%$ lower than monocrystalline graphene supported on $\mathrm{SiO}_{2}$. Technologically, this represents an ultrathin transparent heat spreader with a thermal conductivity similar to bulk copper. However, if copper films were scaled to the thickness of our LBL graphene stacks ${ }^{45}$ the increased boundary scattering would likely reduce the thermal conductivity by more than an order of magnitude, indicating graphene vdW solids have superior performance at the single-nanometer thicknesses we report here.

\section{METHODS}

Graphene growth, transfer, and characterization

Polycrystalline graphene films are grown on 1.4 mil copper foils using a 1inch quartz tube low-pressure CVD system. The copper foils are annealed under $\mathrm{Ar} / \mathrm{H}_{2}$ flow for $60 \mathrm{~min}$ at $1000^{\circ} \mathrm{C}$ prior to graphene growth, which occurs under $\mathrm{CH}_{4}$ and $\mathrm{H}_{2}$ flow at $1000^{\circ} \mathrm{C}$ for $20 \mathrm{~min}$ at a pressure of $\sim 500$ mTorr. Transfer of the graphene films is performed by coating one side of the copper foil with a bilayer of $495 \mathrm{~K}$ and $950 \mathrm{~K} \mathrm{PMMA}$. Graphene films on the opposite side of the copper foil are removed by $\mathrm{O}_{2}$ plasma etching and the copper foil is etched overnight in CE-100 purchased from Transene Corporation. The PMMA-graphene film is cleaned in a $10 \% \mathrm{HCl}$ in DI water solution to remove residual metal particles and rinsed again in DI water 
prior to transferring the film to the receiving substrates. The PMMA is removed in a 1:1 mixture of methylene chloride to methanol for $20 \mathrm{~min}$ and the samples are then annealed in the quartz tube furnace at $400^{\circ} \mathrm{C}$ under $\mathrm{Ar}$ and $\mathrm{H}_{2}$ flow to remove residual PMMA. Transmittance measurements are performed using a Varian CARY 5 G system photospectrometer. Raman measurements are performed using a scanning confocal Renishaw Raman microsope (inVia and WiRE 3.2 software).

\section{Thermometry platforms}

Suspended thermometry platforms were fabricated on a dual-side polished silicon wafer $\left(\sim 300 \mu \mathrm{m}\right.$ thick). A thin layer of $\mathrm{Al}_{2} \mathrm{O}_{3}(\sim 5 \mathrm{~nm})$ is deposited by atomic layer deposition followed by deposition of low-stress $\mathrm{Si}_{3} \mathrm{~N}_{3.3}$ films by plasma enhanced CVD in a mixed frequency mode. The $\mathrm{Al}_{2} \mathrm{O}_{3}$ layer acts as an etch stop for a BOSCH 2 through-wafer etch. Graphene is transferred to the $\mathrm{Si}_{3} \mathrm{~N}_{3.3} / \mathrm{Al}_{2} \mathrm{O}_{3} / \mathrm{Si}$ substrates as previously described, and standard photolithographic techniques are used to pattern the metal electrodes and graphene samples. The graphene is etched from underneath the electrode patterns using an $\mathrm{O}_{2}$ plasma, followed by electron-beam evaporation of $20 \mathrm{~nm} \mathrm{SiO}, 5 \mathrm{~nm}$ of Ti, and $30 \mathrm{~nm}$ of Pd. The thickness of metal contact pads and metal spacers are further increased to $\sim 100 \mathrm{~nm}$ by photolithography and e-beam evaporation, in order to allow for easier wire bonding and to provide a spacer between the device active area and the carrier wafer (Supplementary Information). A final photolithography step is used to align backside etch windows to topside features and through-wafer etching is accomplished using a $\mathrm{BOSCH} 2$ process in an induced coupled plasma reactive ion etcher. Photoresists are removed in Remover PG at $80^{\circ} \mathrm{C}$ following through-wafer etching. Importantly, graphene films are protected throughout all photolithography process steps by a thin layer of PMGI SF5, a PMMA derivative. Samples for cross-plane thermal measurements are fabricated by transferring graphene films as described above, to $\mathrm{SiO}_{2} / \mathrm{Si}(\sim 90 \mathrm{~nm} / 0.5 \mathrm{~mm})$ substrates followed by shadow mask evaporation of $\sim 80 \mathrm{~nm}$ thick Al disks with varying radii between 40 and $125 \mu \mathrm{m}$. Graphene thickness and effective grain size are evaluated with Raman spectroscopy using a $633 \mathrm{~nm}$ laser. The $\mathrm{Si}_{3} \mathrm{~N}_{3.3}$ stoichiometry is measured by X-ray photoelectron spectroscopy.

\section{Electrical and thermal measurement}

In-plane: The heater and sensors of the suspended membrane devices are wirebonded to a KYOCERA leaded ceramic chip carrier, prior to being placed in a Janis vacuum probe station for measurements. The probe station is capable of reaching vacuum levels down to $10^{-6}$ Torr and the ambient temperature is controlled with a Lakeshore model 377 temperature controller and liquid nitrogen cooling. Prior to all measurements the device is annealed for $\approx 8 \mathrm{~h}$ in vacuum at $450 \mathrm{~K}$ to stabilize the resistance of all the metal electrodes. The heater and sensor resistances are calibrated as a function of temperature from $80 \mathrm{~K}$ to $450 \mathrm{~K}$. This is done using a 4-point Delta Mode technique and the Keithley 6221/2182A current source and nanovoltmeter combo. Current is applied to the heater using a Keithley $4200-S C S$ and heater power and resistance are monitored with a 4-point current-voltage measurement. The dependence of the sensor resistance as a function of heater power is monitored by a 4-point Delta Mode technique.

Cross plane: The cross-plane thermal measurement is done by timedomain thermoreflectance (TDTR) using a Ti-sapphire laser that operates at a wavelength near $785 \mathrm{~nm}$. All measurements are performed at room temperature. We use a double modulation technique with the pump beam modulated at $9.8 \mathrm{MHz}$ and the probe beam modulated at $200 \mathrm{~Hz}$ to improve the signal-to-noise and suppress background created by a diffusely scattered pump light. To a good approximation at high modulation frequencies, the in-phase signal of the lock-in amplifier $V_{\text {in }}(t)$ is proportional to the time-domain response of the sample, i.e., the temperature excursion created by each pump optical pulse. The out-ofphase signal $V_{\text {out }}(t)$ is mostly determined by the imaginary part of the frequency domain response at the modulation frequency and is approximately independent of delay time. Comparing $V_{\text {in }} / V_{\text {out }}$ of measurement and of thermal modeling, we determine the cross-plane thermal conductance of the $\mathrm{Al} / \mathrm{N}$ layers of graphene $/ \mathrm{SiO}_{2}$ stack $\left(G_{\perp}\right)$. A complete description of the analysis of TDTR data and the interpretation of $V_{\text {in }}$ and $V_{\text {out }}$ can be found in ref. ${ }^{42}$.

\section{STEM characterization}

The cross-section sample was prepared by lift-out technique using a FEI DB-235 focused-ion beam (FIB). The STEM images and EELS spectra were recorded using a JEOL 2200FS equipped with a CEOS probe corrector at $200 \mathrm{kV}$. Both BF and HAADF detectors were used for recording.

\section{DATA AVAILABILITY}

The data that support the findings of this study are available from the corresponding authors upon reasonable request.

\section{ACKNOWLEDGEMENTS}

We thank M.-H. Bae and J.D. Wood for assistance with the experimental setup and optimization of graphene growth and transfer. Experiments were carried out in part in the Frederick Seitz Materials Research Laboratory at the University of Illinois. This work was sponsored by the National Science Foundation (NSF) Graduate Research Fellowship and the National Defense Science and Engineering Graduate Fellowship (D.E. and S.N.D). D.E. acknowledges career development support from Institutional Development Awards (IDeA) from the National Institute of General Medical Sciences of the National Institutes of Health under Grants \#P20GM103408 and P20GM109095. J.L. acknowledges support from the National Research Foundation of Korea (NRF) grants funded by the Ministry of Science and ICT (NRF-2017R1A2B3009610 and NRF2017R1A4A1015564). F.L. and E.P. acknowledge support from the NSF EFRI 2-DARE program under grant \#1542883.

\section{AUTHOR CONTRIBUTIONS}

D.E. and E.P. conceived the experimental design. D.E. fabricated and measured inplane thermal measurement samples with assistance from S.N.D., J.L., W.P.K., and J.A. R. Z.L. performed finite element modeling, analytical modeling, and uncertainty analysis. G.-M.C. and D.G.C. performed cross-plane thermal measurements and analysis. Y.M. and J.-M.Z. performed TEM imaging and analysis. A.S. performed NEGF calculations. F.L., N.C.W., R.T.H. aided in sample fabrication and materials characterization. All authors made substantial contributions to data acquisition, analysis, and interpretation. All authors discussed results and co-wrote the final manuscript.

\section{ADDITIONAL INFORMATION}

Supplementary information accompanies the paper on the npj $2 D$ Materials and Applications website (https://doi.org/10.1038/s41699-019-0092-8).

Competing interests: The authors declare no competing interests.

Publisher's note: Springer Nature remains neutral with regard to jurisdictional claims in published maps and institutional affiliations.

\section{REFERENCES}

1. Geim, A. \& Grigorieva, I. Van der Waals heterostructures. Nature 499, 419-425 (2013).

2. Novoselov, K. et al. Two-dimensional atomic crystals. Proc. Natl Acad. Sci. USA 102, 10451-10453 (2005).

3. Liu, H. et al. Phosphorene: An unexplored 2D semiconductor with a high hole mobility. ACS Nano 8, 4033-4041 (2014).

4. Vogt, P. et al. Silicene: Compelling experimental evidence for graphene like twodimensional silicon. Phys. Rev. Lett. 108, 155501 (2012).

5. Sutter, P., Lahiri, J., Albrecht, P. \& Sutter, E. Chemical vapor deposition and etching of high-quality monolayer hexagonal boron nitride films. ACS Nano 5, 7303-7309 (2011).

6. Wang, Q. H., Kalantar-Zadeh, K., Kis, A., Coleman, J. N. \& Strano, M. S. Electronics and optoelectronics of two-dimensional transition metal dichalcogenides. Nat. Nanotechnol. 7, 699-712 (2012).

7. Britnell, L. et al. Field-effect tunneling transistor based on vertical graphene heterostructures. Science 335, 947-950 (2012).

8. Gao, G. et al. Artificially stacked atomic layers: Toward new van der Waals solids. Nano Lett. 12, 3518-3525 (2012).

9. Georgiou, T. et al. Vertical field-effect transistor based on graphene-WS $\mathrm{S}_{2}$ heterostructures for flexible and transparent electronics. Nat. Nanotechnol 8, 100-103 (2012).

10. Furchi, M. M., Pospischil, A., Libisch, F., Burgdörfer, J. \& Mueller, T. Photovoltaic effect in an electrically tunable van der Waals heterojunction. Nano Lett. 14, 4785-4791 (2014).

11. Zhang, W. et al. Ultrahigh-gain photodetectors based on atomically thin graphene- $\mathrm{MoS}_{2}$ heterostructures. Sci. Rep. 4, 3826 (2014). 
12. Cahill, D. G. et al. Extremes of heat conduction-pushing the boundaries of the thermal conductivity of materials. MRS Bull. 37, 855-863 (2012).

13. Pop, E., Varshney, V. \& Roy, A. K. Thermal properties of graphene: Fundamentals and applications. MRS Bull. 37, 1273-1281 (2012).

14. Xu, Y., Li, Z. \& Duan, W. Thermal and thermoelectric properties of graphene. Small 10, 2182-2199 (2014)

15. Seol, J. H. et al. Two-dimensional phonon transport in supported graphene. Science 328, 213-216 (2010).

16. Serov, A. Y., Ong, Z.-Y. \& Pop, E. Effect of grain boundaries on thermal transport in graphene. Appl. Phys. Lett. 102, 033104 (2013).

17. Balandin, A. A. Thermal properties of graphene and nanostructured carbon materials. Nat. Mater. 10, 569-581 (2011).

18. Chen, S. et al. Raman measurements of thermal transport in suspended monolayer graphene of variable sizes in vacuum and gaseous environments. ACS Nano 5, 321-328 (2011).

19. Chen, S. et al. Thermal conductivity of isotopically modified graphene. Nat. Mater. 11, 203-207 (2012)

20. Zhao, W., Chen, W., Yue, Y. \& Wu, S. In-situ two-step Raman thermometry for thermal characterization of monolayer graphene interface material. Appl. Therm. Eng. 113, 481-489 (2017).

21. Banerjee, S. et al. Electrochemistry at the edge of a single graphene layer in a nanopore. ACS Nano 7, 834-843 (2013).

22. Kim, R.-H. et al. Stretchable, transparent graphene interconnects for arrays of microscale inorganic light emitting diodes on rubber substrates. Nano Lett. 11 3881-3886 (2011).

23. Li, X. et al. Large-area synthesis of high-quality and uniform graphene films on copper foils. Science 324, 1312-1314 (2009).

24. Salehi-Khojin, A. et al. Polycrystalline graphene ribbons as chemiresistors. Adv. Mater. 24, 53-57 (2012).

25. Nair, R. R. et al. Fine structure constant defines visual transparency of graphene. Science 320, 1308 (2008).

26. Cançado, L. G. et al. General equation for the determination of the crystallite size $L_{a}$ of nanographite by Raman spectroscopy. Appl. Phys. Lett. 88, 163106 (2006).

27. Jorio, A., Dresselhaus, M., Saito, R. \& Dresselhaus, G. F. Raman Spectroscopy in Graphene Related Systems. (Wiley, Hoboken, 2011).

28. Zhu, W. et al. Structure and electronic transport in graphene wrinkles. Nano Lett 12, 3431-3436 (2012)

29. Kim, K. et al. Grain boundary mapping in polycrystalline graphene. ACS Nano $\mathbf{5}$, 2142-2146 (2011).

30. Graf, D. et al. Spatially resolved Raman spectroscopy of single-and few-layer graphene. Nano Lett. 7, 238-242 (2007).

31. Kumar, K., Kim, Y.-S. \& Yang, E.-H. The influence of thermal annealing to remove polymeric residue on the electronic doping and morphological characteristics of graphene. Carbon 65, 35-45 (2013).

32. Huang, P. Y. et al. Grains and grain boundaries in single-layer graphene atomic patchwork quilts. Nature 469, 389 (2011).
33. Bae, M.-H. et al. Ballistic to diffusive crossover of heat flow in graphene ribbons Nat. Commun. 4, 1734 (2013).

34. Li, Z., Bae, M.-H. \& Pop, E. Substrate-supported thermometry platform for nanomaterials like graphene, nanotubes, and nanowires. Appl. Phys. Lett. 105, 023107 (2014).

35. Miguel Muñoz, R. et al. Thermal transport across graphene step junctions. $2 D$ Mater. 6, 011005 (2019)

36. Sadeghi, M. M., Jo, I. \& Shi, L. Phonon-interface scattering in multilayer graphene on an amorphous support. Proc. Natl Acad. Sci. USA 110, 16321-16326 (2013).

37. Koh, Y. K., Bae, M.-H., Cahill, D. G. \& Pop, E. Heat conduction across monolayer and few-layer graphenes. Nano Lett. 10, 4363-4368 (2010).

38. Liang, X. et al. Toward clean and crackless transfer of graphene. ACS Nano $\mathbf{5}$ 9144-9153 (2011).

39. Lupina, G. et al. Residual metallic contamination of transferred chemical vapor deposited graphene. ACS Nano 9, 4776-4785 (2015).

40. Pettes, M. T., Jo, I., Yao, Z. \& Shi, L. Influence of polymeric residue on the thermal conductivity of suspended bilayer graphene. Nano Lett. 11, 1195-1200 (2011).

41. Yasaei, P. et al. Bimodal phonon scattering in graphene grain boundaries. Nano Lett. 15, 4532-4540 (2015).

42. Cahill, D. G. Analysis of heat flow in layered structures for time-domain thermoreflectance. Rev. Sci. Instrum. 75, 5119-5122 (2004).

43. Cai, W. et al. Thermal transport in suspended and supported monolayer graphene grown by chemical vapor deposition. Nano Lett. 10, 1645-1651 (2010).

44. Ho, C. Y., Powell, R. W. \& Liley, P. E. Thermal conductivity of the elements. J. Phys. Chem. Ref. Data 1, 279-421 (1972).

45. Li, Z., Rahtu, A. \& Gordon, R. G. Atomic layer deposition of ultrathin copper metal films from a liquid copper(I) amidinate precursor. J. Electrochem. Soc. 153, C787-C794 (2006).

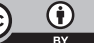

Open Access This article is licensed under a Creative Commons Attribution 4.0 International License, which permits use, sharing, adaptation, distribution and reproduction in any medium or format, as long as you give appropriate credit to the original author(s) and the source, provide a link to the Creative Commons license, and indicate if changes were made. The images or other third party material in this article are included in the article's Creative Commons license, unless indicated otherwise in a credit line to the material. If material is not included in the article's Creative Commons license and your intended use is not permitted by statutory regulation or exceeds the permitted use, you will need to obtain permission directly from the copyright holder. To view a copy of this license, visit http://creativecommons. org/licenses/by/4.0/.

(c) The Author(s) 2019 